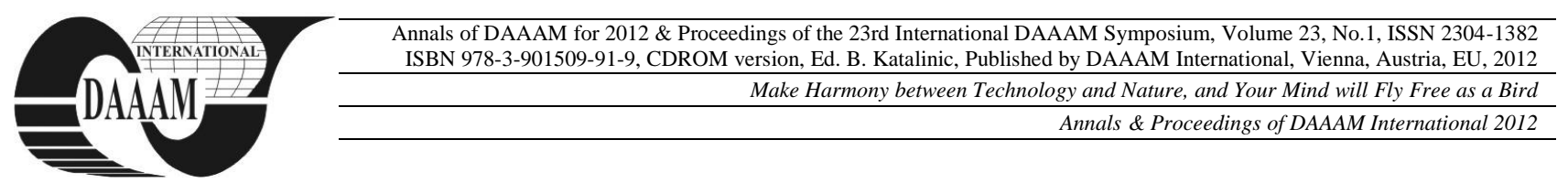

\title{
ANALYSIS OF METHODS FOR CAMERA CALIBRATION IN 3D SCANNING SYSTEMS
}

\author{
SURIANSKY, J[ozef] \& CMARADA, M[ichal]
}

\begin{abstract}
This work aims to compare the two most widely used implementations of camera calibration tools using planar calibration patterns. Comparison of the calibration was performed using the Camera Calibration Toolbox in MATLAB and OpenCV library implementation in C++ using Microsoft Visual Studio 2010. The results of the comparison describe the advantages and disadvantages of each algorithm, the possibility of further extensions and applications. The resulting data are clearly compared in tables and graphs.
\end{abstract}

Keywords: camera calibration toolbox, OpenCV, camera calibration, calibration comparison, reprojection error

\section{INTRODUCTION}

Generally, camera calibration is used to determine intrinsic and extrinsic parameters for camera systems, but it is also used to determine the complete lens distortion model. The intrinsic parameters include focal length, principal point, skew coefficient, distortions and others. The extrinsic parameters can include the rotation matrix and translation vector between the camera coordinate system and the world coordinate system.

Recently, many calibration methods were compared and described. Most of them were based on Tsai, Heikkila \& Silven or Zhang methods [1] [2]. All three methods are based on the pinhole camera model and include radial distortion models. Most of currently used methods use planar point arrays and line-based techniques but some of them also use 3D point arrays.

The method proposed by Tsai assumes that some parameters of the camera are provided by the manufacturer and uses more than eight points per calibration image for computation.

Zhang's method uses a checkerboard pattern, which is placed in front of the camera. At least three different images in various angles and positions must be acquired for the computation of camera calibration model. After the acquisition of the images the algorithms for detection of corners are used and corners of the checkerboard pattern are extracted. These points are used in calculations for camera calibration. This method is also used in OpenCV and Camera calibration.

Our goal was to follow up on this work and offer an overview of the most commonly used tools available to calibrate the cameras for 3D scanning systems. An important factor in the comparison of these tools was the use of open source code and the portability to most commonly used operating systems. These tools must also provide us the opportunity to create robust application which could be used in 3D scanning systems in the process of casting machine parts.

The disadvantage of this solution is the use of $2 \mathrm{D}$ calibration pattern, which usually takes long time. A better solution would be to use $3 \mathrm{D}$ calibration object. This object could be placed in the scanning area and the calibration will be carried out during the scanning process. This approach we would like to implement in our future research.

\section{CAMERA CALIBRATION MODEL}

Calibration of the camera allows us to determine the important parameters that can be used for making measurements in space. This process is called perspective projection [3] and it describes the geometric relationship, which to each three-dimensional point $\mathrm{A}[\mathrm{X}, \mathrm{Y}, \mathrm{Z}]$ assigns two-dimensional point $\mathrm{O}[\mathrm{x}, \mathrm{y}]$ in the image coordinate system (Fig. 1).

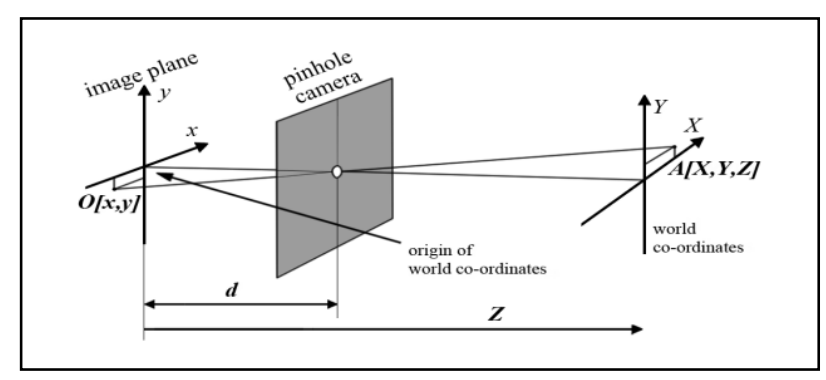

Fig. 1. Perspective Projection [3]

Assume that the image " $x, y$ " plane is parallel to the global coordinates " $\mathrm{X}, \mathrm{Y}$ " plane, and the optical axis is parallel to the $\mathrm{Z}$ axis. Next, assume that the focal point is located in the pinhole camera (PC - Pinhole Camera) at a distance of "d" (focal distance) from the image plane. Then any $3 \mathrm{D}$ point $\mathrm{A}[\mathrm{X}, \mathrm{Y}, \mathrm{Z}]$ is projected in the image plane at point $\mathrm{O}[\mathrm{x}, \mathrm{y}]$ according to a mathematical formula (1)[3]:

$$
x=\frac{-d \cdot X}{Z-d}, y=\frac{-d \cdot Y}{Z-d}
$$

\section{CAMERA CALIBRATION}

Before the calibration it is necessary to adjust the 3D scanning system. It must be set to cover the whole desired scanning area. This means determining the position of the camera, zoom and focus on the scanning surface. After the calibration it is no longer possible to 
manipulate this system and change its parameters, otherwise it would be necessary to perform the calibration again.

It is necessary to use appropriate calibration objects, patterns or shapes. These patterns are then used in the calculations of intrinsic and extrinsic parameters of the system based on images acquired by the digital camera. The most commonly used is the checkerboard pattern Fig. 2 which is used also in camera calibration toolbox for MATLAB and OpenCV. This pattern is printed on a solid flat surface and has predefined dimensions and parameters (for example. checkerboard pattern of $6 \times 6$ squares and 20x20mm size each).

There are also many other calibration pattern types Fig. 2 that are widely used. Most of them are planarbased but some are also represented in 3D space.

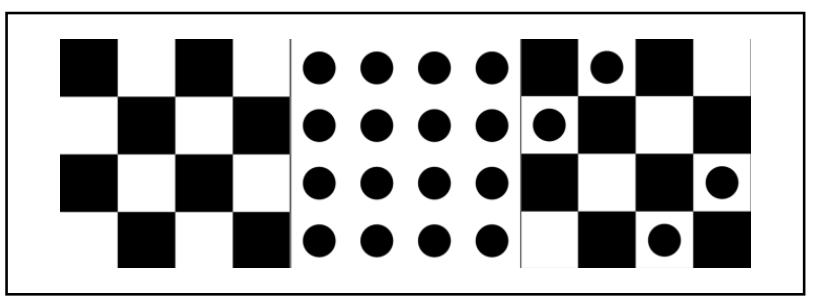

Fig. 2. Calibration patterns example

\subsection{Camera Calibration Toolbox for Matlab}

Camera calibration toolbox [4] is a robust tool in the mathematical computing environment of MATLAB [5], which allows us to calibrate the cameras of the optical based 3D scanning systems. Its advantage is the use of open source code, which contributes to the implementation of its functionalities in various 3D scanning systems such as projector-camera or cameracamera based systems. It represents a fundamental building block that can be extended to cover the overall calibration of the 3D scanning system.

It provides many tools Fig. 3 to analyze the results of calibration, which makes it possible to suppress calibration errors to a minimum. On Fig. 3 we can see the distribution of reprojection errors in both $\mathrm{x}$ and $\mathrm{y}$ axis. Crosses represent the intersection points of calibration patterns and their distance from the center is equal to the reprojection error for the particular point. Values of both axes and also the values of reprojection error are given in pixels.

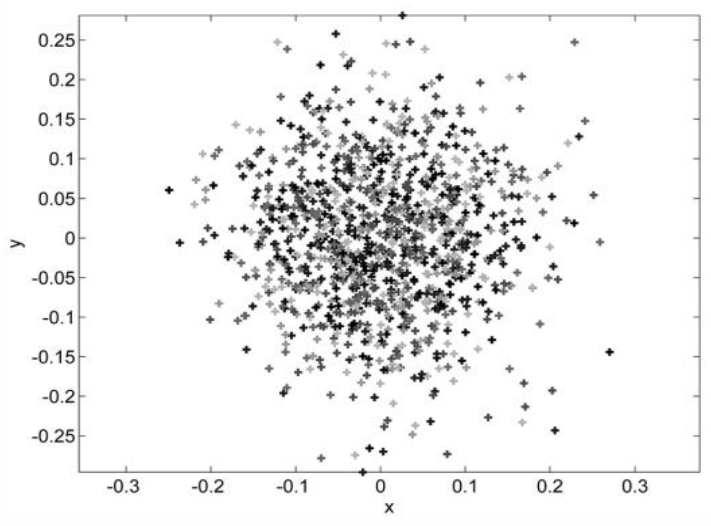

Fig. 3. Reprojection error Analysis
It also gives us a visualization of the results of extrinsic parameters Fig. 4, which is necessary for subsequent calculations of distances. On this figure you can see the position of every calibration pattern obtained from the calibration images acquired by the digital camera. The position of the camera was fixed and the position and the angle of calibration pattern were changed for every captured image.

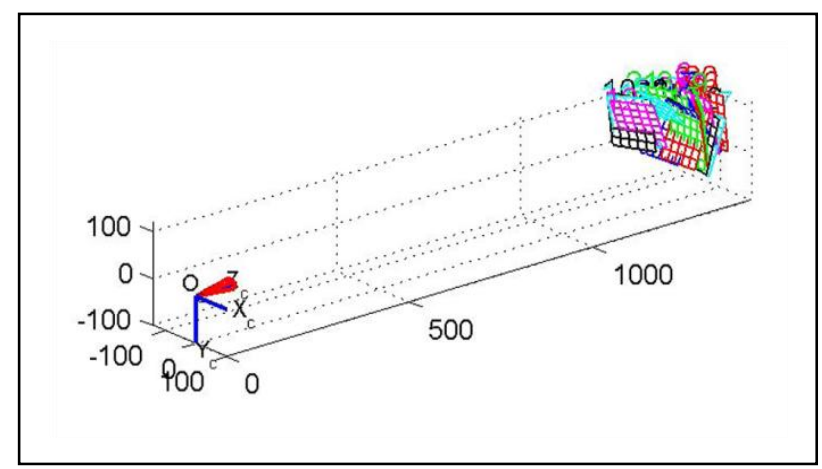

Fig. 4. Extrinsic parameters - Camera centered

This toolbox gives us also the tools to visualize the distortion model of the optical system of lenses, mainly tangential, radial and complete camera distortion model Fig. 5. These results are used to eliminate the distortion of acquired images from the digital camera for achieving better results.

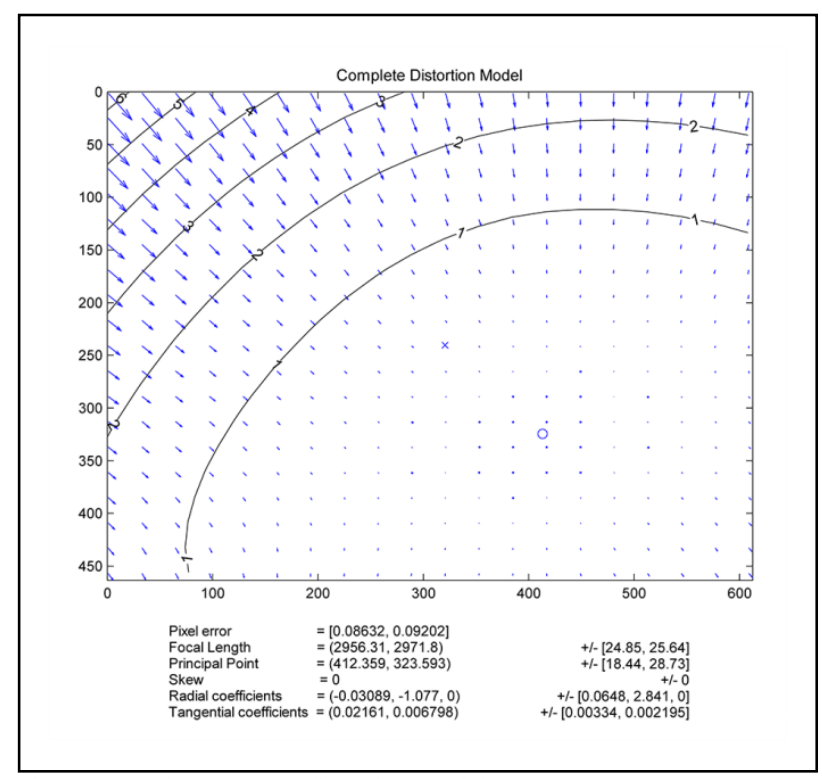

Fig. 5. Complete Camera distortion model

The main disadvantage of camera calibration toolbox is the need of carrying out certain steps manually (especially in comparison with fully automated calibration method via OpenCV), thereby extending the time of calibration. The most time consuming is the determination of borders of calibration pattern. Because in every calibration image (there are normally more than 20 images) it is necessary to define four border points Fig. 6. Further it is necessary to manually enter some configuration parameters and also confirm and execute individual calibration steps.

The advantage however is that this system is open and it is possible to implement additional features such as 
the automation of individual calibration steps, multiple digital camera calibration or calibration of projectorcamera based 3D scanning systems. There are also many tools that implement basic functionalities of Camera calibration toolbox and add various functionalities or optimizations for this toolbox.

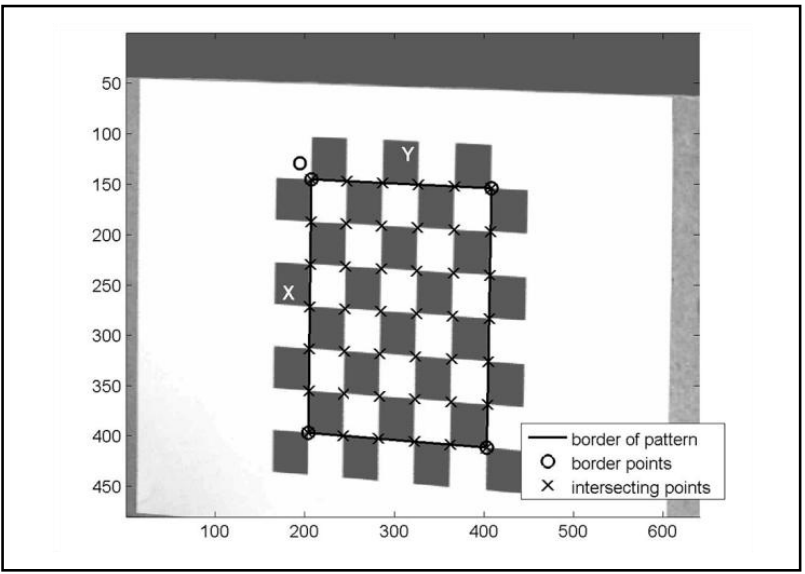

Fig. 6. Calibration image example

\subsection{OpenCV v4.2.1 calibration}

OpenCV (Open Computer vision) is a set of open source libraries [6] [7] for applications in computer vision systems. Currently these libraries consist of more than 2500 optimized algorithms and are available for several programming languages (c, c++, python, and java) and also for several operating systems (Windows, Mac, Linux, Android). These libraries are freely available for academic and commercial sectors. These libraries are open source, therefore the developers are able to modify and adapt them to the requirements of individual computer vision systems.

Unlike Camera calibration toolbox for Matlab this calibration method is fully automated and can be implemented also in real-time computer vision systems. It contains algorithms for automatic detection of the calibration pattern with the detection of inner intersection points of the checkerboard Fig. 7. With this method it is necessary to create a configuration file in XML format, which defines the basic parameters of the calibration (type of pattern, size and distance of objects in the pattern, image acquisition devices, the number of squares of the checkerboard pattern, etc.)

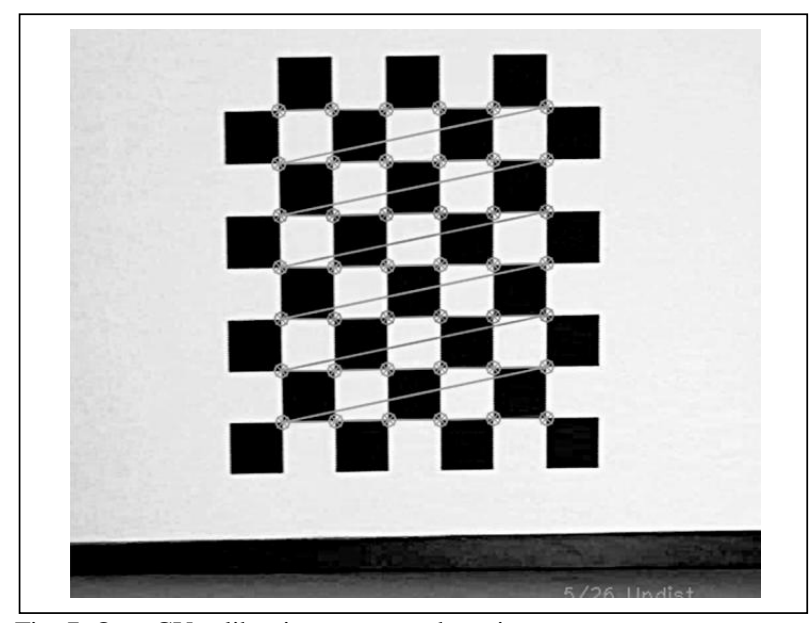

Fig. 7. OpenCV calibration - pattern detection
Disadvantage compared to the previous calibration technique is the need to create custom calibration system based on available OpenCV libraries and knowledge of a particular programming language and development environment.

On the other hand, the use of these libraries has also many advantages. For example we can build our own application for 3D scanning based on these libraries. Such application will be easy to port to different operating systems environments and implement the required functionality (overall calibration of 3D scanning system based on projector-camera calibration).

\section{EXPERIMENT}

To verify the calibration results of each method an experiment was established. The basis for this was the construction of five sets of calibration data. Each set contains 26 calibration images and for each of these sets a calibration was performed using both calibration methods. Calibration results were then used in 3D scanning system and scanning of real 3D models of parts. The model consisted of several scans, which were joined together at the end of the scanning process.

In order to assess the individual results it was necessary to choose the parameter for comparison of different methods. We have decided to use reprojection error as the parameter for comparison. This parameter determines the deviation of the measured point from the projection point. Reprojection error is given in pixels. The calibration is most accurate when this number approaches zero.

\subsection{Results}

After the calibration of all five sets of calibration data, reprojection errors were determined for both methods. The average value of reprojection error was also determined and times necessary to perform calibrations were measured. The results are shown in Table 1.

\begin{tabular}{|c|c|c|c|c|}
\hline \multirow{2}{*}{ 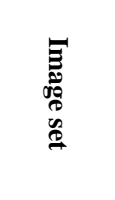 } & \multicolumn{2}{|c|}{$\begin{array}{c}\text { Reprojection error } \\
\text { [pixel] }\end{array}$} & \multicolumn{2}{|c|}{$\underset{[s]}{\text { Time }}$} \\
\hline & $\begin{array}{c}\text { Camera } \\
\text { calibration } \\
\text { toolbox }\end{array}$ & $\begin{array}{c}\text { OpenCV } \\
\text { calibration }\end{array}$ & $\begin{array}{c}\text { Camera } \\
\text { calibration } \\
\text { toolbox }\end{array}$ & $\begin{array}{c}\text { OpenCV } \\
\text { calibration }\end{array}$ \\
\hline 1. set & 0,08917 & 0,16394 & 335,72 & 23,39 \\
\hline 2. set & 0,08548 & 0,15492 & 260,17 & 24,27 \\
\hline 3. set & 0,08747 & 0,16029 & 364,32 & 23,31 \\
\hline 4. set & 0,08009 & 0,15690 & 286,14 & 24,51 \\
\hline 5. set & 0,07894 & 0,15316 & 279,86 & 23,68 \\
\hline Average & 0,08423 & 0,15784 & 305,242 & 23,83 \\
\hline
\end{tabular}

Tab. 1. Comparison of calibration tools

The measurement results show that more accurate calibration can be achieved by Camera calibration toolbox for Matlab. Therefore, this method should be used mainly for laboratory experiments, measurements and analyzes. 
Reprojection error for the OpenCV calibration reached in comparison with the previous solution nearly doubled values. However, thanks to its speed and simplicity, it is advised to use it in industrial and manufacturing applications.

For a better understanding the results are shown also in visual form on Fig. 8 .

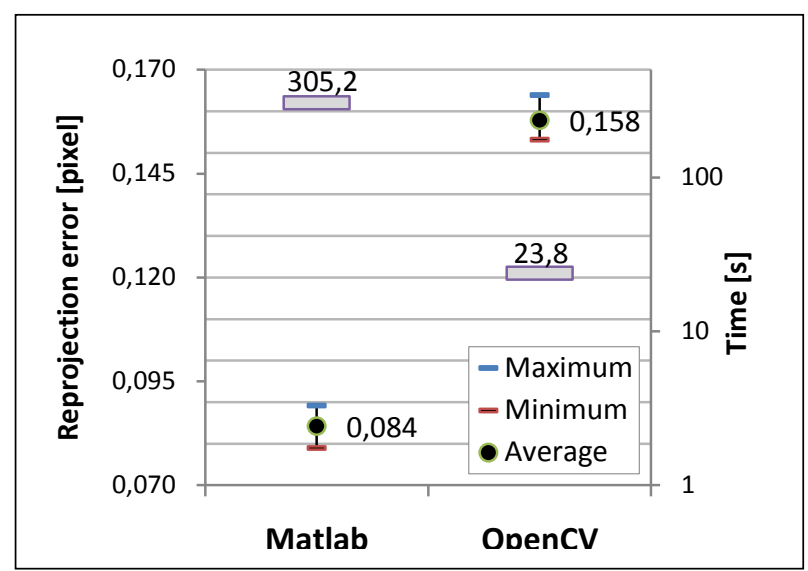

Fig. 8. Reprojection error and time comparison

\section{CONCLUSION}

By comparing different methods of calibration advantages and disadvantages of these methods have been found. Also the time required for calibration and accuracy of the calibration methods was measured. These results were evaluated based on multiple criteria such as accuracy, applicability in industrial production, the possibility of future extension and others.

Calibration using the "Camera calibration toolbox" is mostly suited for applications which require higher precision calibration but which does not have great demands on its speed. On the other hand, calibration using OpenCV libraries is ideal for applications requiring high speed calibration with sufficient accuracy. Both of these methods are open source based and can be freely implemented and improved.

Using our experimental system for 3D optical scanning (resolution of the camera 640x480 pixels and projector resolution of $800 \times 600$ pixels) we achieved a resolution of $0.44 \mathrm{~mm}$ for this system.

This system was used for making test scans of casted parts Fig. 9.

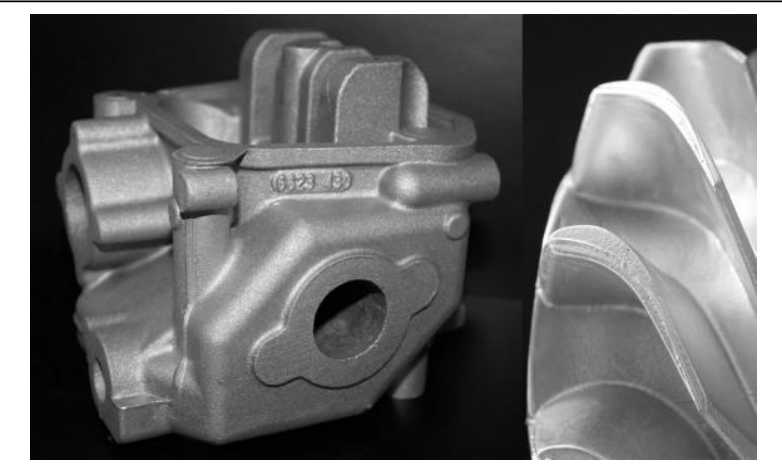

Fig. 9. Example of casted parts
Partial scans were then joined together to create real 3D model of this part Fig. 10.

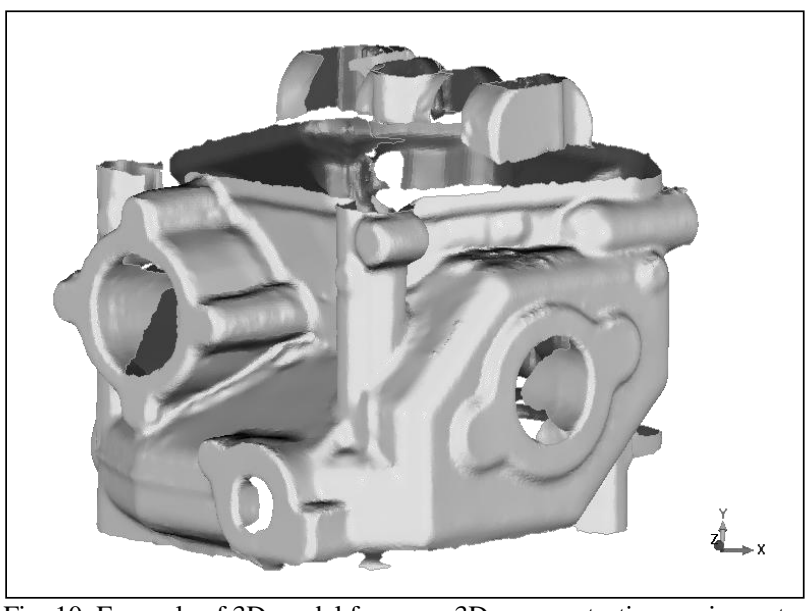

Fig. 10. Example of 3D model from our 3D scanner testing equipment

The castings that are used in our experiments are the production of company ILLICHMAN CASTALLOY s.r.o. Slovakia. The part measures approximately $135 \mathrm{~mm} \times 120 \mathrm{~mm} \times 110 \mathrm{~mm}$. It is cast from aluminum alloy and prior to the scanning process the surface of this part was not treated in any way. Because of this there were some problems with reflections on metallic surface. Analysis of suppression of these reflections is also a part of our work and will be performed in the next stages of our research.

Our next step will be improving the 3D scanning system to achieve resolution of better than $0.1 \mathrm{~mm}$. The aim is to create fast, robust, efficient and low price 3D scanning system with sufficient accuracy for inspection and measurement of quality of casting machine parts.

By combining more modern equipment (Full HD camera and digital projector) and the calibration tools we plan to upgrade our 3D scanning system based on our own procedures and algorithms with projector-camera calibration.

\section{REFERENCES}

[1] Remondino, F. \& Fraser, C. (2006). Digital camera calibration methods: considerations and comparisons, Avaible from: http://www.isprs.org/proceedings/XXXVI/part5/paper/ REMO_616.pdf Accessed: 2012-06-18

[2] Zollner, H. \& Sablatnig R. (rok). Comparison of Methods for Geometric Camera Calibration using Planar Calibration Targets, Avaible from: http://www.caa.tuwien.ac.at/cvl/people/sab/ papers/oagm04b.pdf Accessed: 2012-06-18

[3] Regtien, P.P.L.; Heijden, F.; Korsten, M. J. \& Otthius, W. (2004) Measurement Science For Engineers, Kogan Page Science, ISBN: 9781903996584, London UK

[4] http://www.vision.caltech.edu/bouguetj/calib_doc/,

(2012). Camera Calibration Toolbox for Matlab, Accessed on: 2012-0712

[5] http://www.mathworks.com/help/techdoc/, (2012). Matlab: Product Documentation, Accessed on: 2012-07-12

[6] Bradski, G. \& Kaebler, A. (2008) Learning OpenCV: Computer Vision with the OpenCV Library, O’Reilly Media, Inc., ISBN: 9780596516130, Sebastopol CA

[7] Laganière, R. (2011) OpenCV 2 Computer Vision Application Programming Cookbook, Packt Publishing Ltd., ISBN: 9781849513241, Birmingham UK 